\title{
FOSFITOS APLICADOS EM PÓS-COLHEITA REDUZEM O MOFO-AZUL EM MAÇÃS 'FUJI' E 'GALA'
}

\author{
LUIZ EDUARDO BASSAY BLUM ${ }^{2}$, CASSANDRO VIDAL TALAMINI DO AMARANTE ${ }^{3}$, ALEXANDRE DEZANET ${ }^{4}$, \\ EDUARDO BARRETO DE LIMA ${ }^{4}$, PEDRO HACK NETO ${ }^{4}$, ROBERTA DUARTE Á VILA $^{4}$, VANUSA SIEGA $^{4}$
}

RESUMO - O mofo-azul (Penicillium expansum) é uma podridão pós-colheita comum em maçãs (Malus domestica). O uso de substâncias menos ofensivas ao ambiente, como o fosfito (ácido fosforoso), é uma das alternativas de controle dessa doença. Visouse, então, a avaliar neste estudo a eficiência de fosfito- $\mathrm{K}\left(40 \%\right.$ de $\mathrm{P}_{2} \mathrm{O}_{5}$ e $30 \%$ de $\left.\mathrm{K}_{2} \mathrm{O}\right)$ e fosfito-CaB $\left(10,7 \%\right.$ de $\mathrm{P}_{2} \mathrm{O}_{5}, 3,89 \%$ de $\mathrm{Ca}$ e $0,5 \%$ de B) no controle do mofo-azul em maçãs 'Fuji' e 'Gala'. Os testes foram delineados em blocos ao acaso com seis repetições de 20 frutos cada. Os frutos foram desinfestados em hipoclorito de $\mathrm{Na}(1 \%)$ por três minutos, lavados em água esterilizada, feridos (1 mm de diâmetro e de profundidade) com agulha em quatro pontos eqüidistantes, imersos nos tratamentos por 15 minutos e armazenados a $15-20^{\circ} \mathrm{C}$. Nas suspensões contaminadas com Penicillium expansum $\left(10^{2}\right.$ conídios. $\left.\mathrm{mL}^{-1}\right)$, foram adicionadas as seguintes substâncias: benomil (150 mg. $\left.\mathrm{L}^{-1}\right)$, fosfito-K $\left(0,5\right.$ a 1,5 mL.L $\left.{ }^{-1}\right)$ e fosfito-CaB $\left(1,5\right.$ a 3,0 mL.L $\left.{ }^{-1}\right)$. As maçãs 'Fuji' e 'Gala' imersas em água com fosfito$\mathrm{CaB}\left(1,5 \mathrm{~mL} \cdot \mathrm{L}^{-1}\right)$, fosfito-K $\left(0,5-1,5 \mathrm{~mL} \cdot \mathrm{L}^{-1}\right)$ ou benomil $\left(150 \mathrm{mg} \cdot \mathrm{L}^{-1}\right)$ foram menos afetadas pelo mofo-azul. A aplicação de fosfito-K (1,5 $\left.\mathrm{mL} . \mathrm{L}^{-1}\right)$ ou benomil (150 mg. $\left.\mathrm{L}^{-1}\right)$ nos frutos foi mais eficiente do que os demais tratamentos no controle do mofo-azul.

Termos para indexação: Malus domestica Borkh., Penicillium expansum, ácido fosfônico, podridão-mole.

\section{POSTHARVEST APPLICATION OF PHOSPHITES REDUCES BLUE MOLD ON 'FUJI'AND 'GALA'APPLES}

\begin{abstract}
Blue mold (Penicillium expansum) is a common postharvest disease of apples (Malus domestica). Applications of less hazardous substances to the environment, such as phosphite (phosphonic acid), is an alternative to the control of blue mold. The main goal of this study was to evaluate the efficiency of phosphite- $\mathrm{K}\left(40 \% \mathrm{P}_{2} \mathrm{O}_{5}\right.$ and $\left.30 \% \mathrm{~K}_{2} \mathrm{O}\right)$ and phosphite-CaB $\left(10.7 \% \mathrm{P}_{2} \mathrm{O}_{5}, 3.89 \%\right.$ $\mathrm{Ca}$, and $0.5 \% \mathrm{~B}$ ) for the control of blue mold on 'Fuji' and 'Gala' apples. Tests were designed in randomized blocks with six replications of 20 fruits. Fruits were decontaminated with Na hypochlorite (1\%) for three minutes, washed with sterilized water, needle wounded (with a diameter and deepness of $1 \mathrm{~mm}$ ) in four equidistant points, dipped into treatments for 15 minutes, and then stored at $15-20^{\circ} \mathrm{C}$. The following substances were added in suspensions contaminated with Penicillium expansum (at the concentration of $1 \times 10^{2}$ conidia.mL $\mathrm{m}^{-1}$ ): benomyl (at $150 \mathrm{mg} . \mathrm{L}^{-1}$ ), phosphite-K (from 0.5 to $1.5 \mathrm{~mL} \cdot \mathrm{L}^{-1}$ ) and phosphite-CaB (from 1.5 to $3.0 \mathrm{~mL} . \mathrm{L}^{-1}$ ). ' $\mathrm{Fuji}$ ' and 'Gala' apples dipped in water with phosphite-CaB $\left(1.5 \mathrm{~mL} . \mathrm{L}^{-1}\right)$, phosphite-K $\left(0.5-1.5 \mathrm{~mL} . \mathrm{L}^{-1}\right)$ or benomyl $\left(150 \mathrm{mg} . \mathrm{L}^{-1}\right)$ were less affected by the blue mold. Application of phosphite-K $\left(1.5 \mathrm{~mL} . \mathrm{L}^{-1}\right)$ or benomyl were the most effective treatments to control the disease.
\end{abstract}

Index terms: Malus domestica Borkh., Penicillium expansum, phosphonic acid, soft rot.

\section{INTRODUÇÃO}

Penicillium expansum, causador do mofo-azul, é um dos principais responsáveis pelas perdas ocasionadas pelas podridões, em várias regiões produtoras de maçãs (Malus domestica Borkh.). As maçãs 'Fuji' e 'Gala', sob condições favoráveis à doença, são semelhantemente afetadas pelo fungo (Blum et al., 2004). O mofo-azul, antes do uso de câmaras frias com atmosfera controlada (AC) para o armazenamento dos frutos, era responsável por até $90 \%$ das perdas. Ultimamente, essas perdas atingem cerca de $1 \%$ devido à estocagem refrigerada dos frutos em condições de AC (Rosenberger, 1990).

O manejo tradicional do mofo-azul preconiza a aplicação de fungicidas (tiabendazol, benomil e iprodione) nos frutos em pós-colheita. Atualmente, com o objetivo de reduzir a concentração de resíduos tóxicos aos seres humanos e animais, e reduzir os danos ao ambiente, tem-se testado para o tratamento pós-colheita de frutos de pomáceas, o controle biológico (Blum et al., 2005; Lima et al., 2005) e substâncias menos tóxicas, porém de ação fungicida, como é o caso dos derivados do ácido fosforoso (fosfonato) ou o íon fosfito (Brackmann et al., 2004). A ação antifúngica dos fosfitos foi relatada inúmeras vezes contra diferentes patógenos das mais variadas plantas cultivadas. Os fosfitos podem atuar diretamente inibindo o desenvolvimento dos fungos e, também, indiretamente ativando o sistema de defesa da planta hospedeira (McKay et al., 1992; Davis et al., 1994; Speiser et al., 1999; Wild et al., 1998).

Neste trabalho, visou-se a avaliar a eficiência de fosfitos (fosfito-K e fosfito-CaB) no controle pós-colheita do mofo-azul em maçãs 'Gala' e 'Fuji'.

(Trabalho 193-06). Recebido em 09-11-2006. Aceito para publicação em 09-05-2007.

2 Ph.D., Bolsista do CNPq, Universidade de Brasília, Departamento de Fitopatologia, CEP 70910-900, Brasília, DF. E-mail: luizblum@unb.br

${ }^{3}$ Ph.D., Bolsista do CNPq, Centro de Ciências Agroveterinárias (CAV), Universidade do Estado de Santa Catarina (UDESC), Cx. Postal 281, CEP 88520000, Lages, SC. E-mail: amarante@cav.udesc.br

${ }^{4}$ Acadêmicos do Curso de Agronomia, Centro de Ciências Agroveterinárias (CAV), Universidade do Estado de Santa Catarina (UDESC), Cx. Postal 281, CEP 88520-000, Lages, SC 


\section{MATERIAL E MÉTODOS}

Culturas puras de Penicillium expansum foram cultivadas em BDA (batata descascada $200 \mathrm{~g}$, dextrose $20 \mathrm{~g}$, ágar $15 \mathrm{~g}$ e água destilada $1 \mathrm{~L})$ sob temperatura de $20^{\circ} \mathrm{C}\left( \pm 2{ }^{\circ} \mathrm{C}\right)$ durante sete dias. Antes da determinação da quantidade de inóculo do patógeno, os conídios foram extraídos em água esterilizada, e a suspensão obtida foi filtrada em camada dupla de gaze. A determinação da concentração do inóculo do fungo (número de conídios. $\mathrm{mL}^{-1}$ ) foi estimada através de câmara de Neubauer.

Os experimentos foram realizados no Laboratório de Fitopatologia do Centro de Ciências Agroveterinárias da Universidade do Estado de Santa Catarina (UDESC), entre fevereiro de 2001 e abril de 2002.

Os ensaios foram delineados em blocos ao acaso, com seis repetições de 20 frutos. Inicialmente, os frutos das cvs. Gala e Fuji foram desinfestados em hipoclorito de $\mathrm{Na}(1 \%)$ por três minutos e então submetidos a perfurações, com diâmetro e profundidade de aproximadamente $1 \mathrm{~mm}$, na região equatorial, em quatro pontos aproximadamente eqüidistantes (Blum et al., 2004). Em seguida, os frutos foram imersos (15 minutos) nas suspensões com $10^{2}$ conídios. $\mathrm{mL}^{-1}$ ou sem conídios do patógeno, e com as substâncias fungicidas (fosfitos e benomil) testadas.

Os fosfitos usados nos experimentos foram o fosfito-K (com $40 \%$ de $\mathrm{P}_{2} \mathrm{O}_{5}$ e $30 \%$ de $\mathrm{K}_{2} \mathrm{O}$ ) e o fosfito-CaB (com 10,7\% de $\mathrm{P}_{2} \mathrm{O}_{5}, 3,89 \%$ de $\mathrm{Ca}$ e $0,5 \%$ de $\mathrm{B}$ ).

Dois grupos de experimentos em um delineamento em blocos ao acaso foram efetuados: 1$)$ doses crescentes de fosfito$\mathrm{K}\left(0 ; 0,5 ; 1,0\right.$ e 1,5 mL.L $\left.{ }^{-1}\right)$ em maçãs 'Fuji' e ' Gala' e doses crescentes de fosfito-CaB $\left(0 ; 0,75 ; 1,5\right.$ e $\left.3,0 \mathrm{ml} . \mathrm{L}^{-1}\right)$ em maçãs 'Fuji', e 2) tratamentos com benomil (150 mg. $\left.\mathrm{L}^{-1}\right)$, fosfito-K $(1,5$ mL.L $\left.{ }^{-1}\right)$ e fosfito-CaB (1,5 mL.L-1) em maçãs 'Fuji' e 'Gala'. Posteriormente à inoculação, os frutos foram incubados no escuro, a $20^{\circ} \mathrm{C}\left( \pm 2^{\circ} \mathrm{C}\right)$. Após o aparecimento dos sintomas iniciais, a cada 48 horas, foram avaliados a incidência (\%) e o diâmetro das lesões $(\mathrm{mm})$ da doença ocasionadas pelo patógeno.

Análises de regressão foram efetuadas entre as variáveis numéricas, doses dos produtos e quantidade de doença (incidência e diâmetro da lesão da doença) no experimento 1. As médias dos diferentes tratamentos experimentais foram comparadas entre si, através do teste de Tukey $(\mathrm{P}<0,05)$, e quando comprovada a significância dos dados, procedeu-se a análise de variância de entrada dupla $(\mathrm{F}=5 \%$ ) (SigmaStat, versão 2, 1995, Jandel Corporation).

\section{RESULTADOS E DISCUSSÃO}

Os fosfitos (fosfito-K e fosfito-CaB) testados reduziram a quantidade do mofo-azul, de forma similar ao observado com o tratamento tradicional à base de fungicida benomil. Salienta-se que o benomil, atualmente não mais comercializado, pode ser substituído por outros fungicidas tradicionais, tais como o tiabendazol e o iprodione (Blum et al., 2004).
Tratamentos pós-colheita de frutos de maçãs com doses crescentes de fosfito- $\mathrm{K}$ e fosfito-CaB reduziram a incidência e o diâmetro das lesões ocasionadas por P. expansum (Figuras 1AF). Doses entre 1,0 e 1,5 mL. $\mathrm{L}^{-1}$ de fosfito-K reduziram a incidência da doença até níveis entre 0,5 e $5 \%$ em maçãs 'Fuji' e 'Gala' (Figuras 1A e 1C). Fosfito-CaB, em doses entre 1,5 e 3,0 mL.L-1, reduziu a incidência da podridão a valores entre 1 e 4\% em maçã 'Fuji' (Figura 1E). Também, o diâmetro das lesões foi reduzido em doses entre 1,0 e 1,5 mL. $\mathrm{L}^{-1}$ de fosfito-K $(<1 \mathrm{~mm}$ ) (Figuras $1 \mathrm{~B}$ e 1D) e entre 1,5 e $3,0 \mathrm{~mL} \cdot \mathrm{L}^{-1}$ de fosfito- $\mathrm{CaB}(<3 \mathrm{~mm})$ (Figura $1 \mathrm{~F}$ ).

$\mathrm{O}$ ácido fosforoso possui propriedades indutoras de resistência nos vegetais (Wild et al., 1998) e pode também reduzir a esporulação dos patógenos nas plantas, com isso possibilitando a redução na incidência e na severidade das fitomoléstias (Panicker \& Gangadharan, 1999). Wild et al. (1998) mostraram que a concentração de enzimas relacionadas à defesa em maçãs aumentou quando houve tratamento prévio dos frutos com fosfito. Reuveni et al. (2003) relataram a redução na incidência de podridão em maçãs 'Red Delicious', ocasionada por Alternaria alternata (Fr.) Keissler, pela aplicação de fosfito de potássio $(0,3 \%)$. Estes autores comentam que a ação do fosfito envolve a ação direta sobre a germinação dos esporos e pode, indiretamente, atuar através da indução na produção de fitoalexinas nas células dos frutos. Sala et al. (2004) relataram que a aplicação de fosfito de potássio $\left(30 \%\right.$ de $\mathrm{P}_{2} \mathrm{O}_{5}$ e $20 \%$ de $\left.\mathrm{K}_{2} \mathrm{O}\right)$ aumentou a reação de resistência de alguns genótipos de pimentão (Capsicum annuum L.) à Phytophthora capsici Leonian. Estes autores inferem que o fosfito agiu, possivelmente, através da indução da produção de fitoalexinas no hospedeiro e da inibição direta do patógeno. Tais propriedades indutoras de resistência e fungistáticas, relacionadas aos fosfitos, podem culminar com a redução da quantidade de doença.

A adição de fosfito-CaB $\left(1,5 \mathrm{~mL} . \mathrm{L}^{-1}\right)$ ou fosfito-K $\left(1,5 \mathrm{~mL} . \mathrm{L}^{-1}\right)$ em água contaminada com $P$. expansum foi tão eficiente quanto a adição de benomil (150 mg. $\left.\mathrm{L}^{-1}\right)$ na redução do mofo-azul em maçãs 'Fuji' e 'Gala' (Figura 2). Frutos tratados com fosfito K (1,5 mL. $\left.\mathrm{L}^{-1}\right)$ e benomil (150 mg. $\left.\mathrm{L}^{-1}\right)$ foram menos afetados pela doença do que os submetidos aos demais tratamentos.

As doses dos fosfitos [fosfito-CaB $\left(1,5 \mathrm{~mL} . \mathrm{L}^{-1}\right)$ e fosfito$\left.\mathrm{K}\left(1,5 \mathrm{~mL} . \mathrm{L}^{-1}\right)\right]$ usadas neste estudo são inferiores às testadas por Brackmann et al. (2004) [fosfito-CaB (3,0 mL.L $\left.{ }^{-1}\right)$ e fosfito-K $\left.\left(2,5 \mathrm{~mL} \cdot \mathrm{L}^{-1}\right)\right]$, que relataram a redução na incidência de podridões em maçãs 'Fuji', possivelmente ocasionadas por Penicillium, Botrytis ou Rhizopus, pelo uso dos fosfitos. Todavia, tais autores relataram que o fosfito- $\mathrm{CaB}\left(3,0 \mathrm{~mL} . \mathrm{L}^{-1}\right)$ não reduziu significativamente o tamanho das lesões, o que contrasta com os resultados aqui apresentados. Tal diferença pode estar associada a diferenças na metodologia de inoculação do patógeno. No presente estudo, a inoculação foi realizada com uma concentração conhecida de $P$. expansum, enquanto, no estudo efetuado por Brackmann et al. (2004), a concentração de inóculo dos patógenos (Penicillium, Botrytis ou Rhizopus) não foi determinada. 

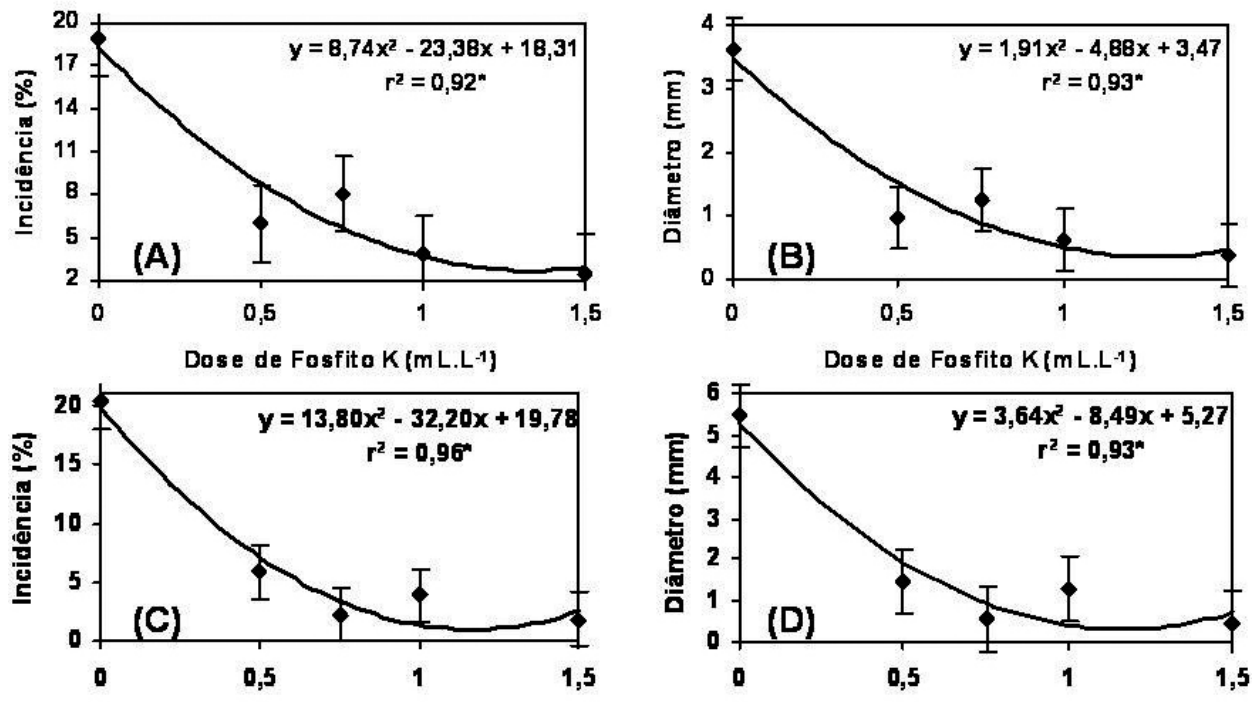

Dose de Fosfito K(mL.L-1)
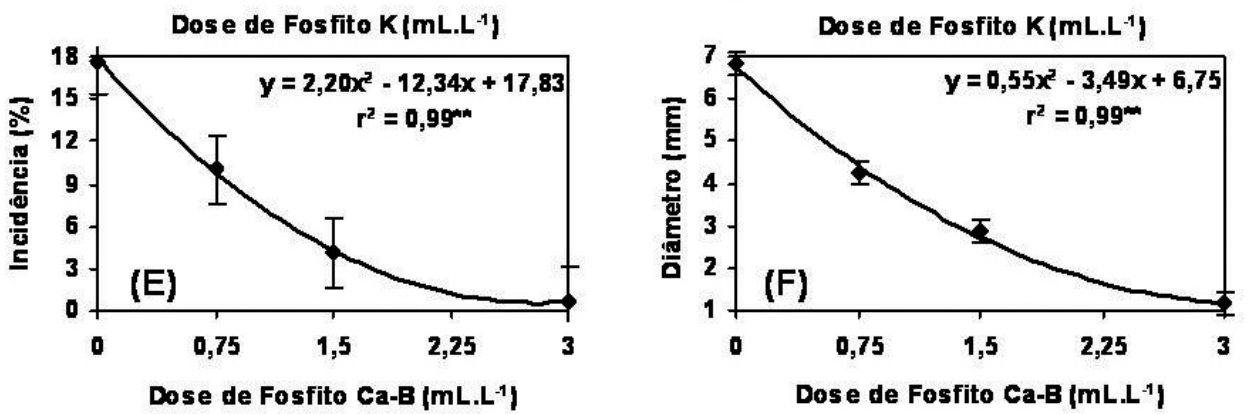

FIGURA 1 - Valores médios de incidência (\%) e diâmetro da lesão (mm), dos seis aos 14 dias após a inoculação de Penicillium expansum, em maçãs 'Fuji' (A e B) e 'Gala' (C e D), tratadas com diferentes doses de fosfito-K, e em maçãs 'Fuji' tratadas com diferentes doses de fosfito-CaB (E e F). A barra vertical em cada ponto corresponde ao erro-padrão estimado. Os símbolos $\left(^{*}\right)$ e $(* *)$ correspondem, respectivamente, à regressão significativa a 5\% e $1 \%$ de probabilidade de erro. Lages-SC, 2001/2002.
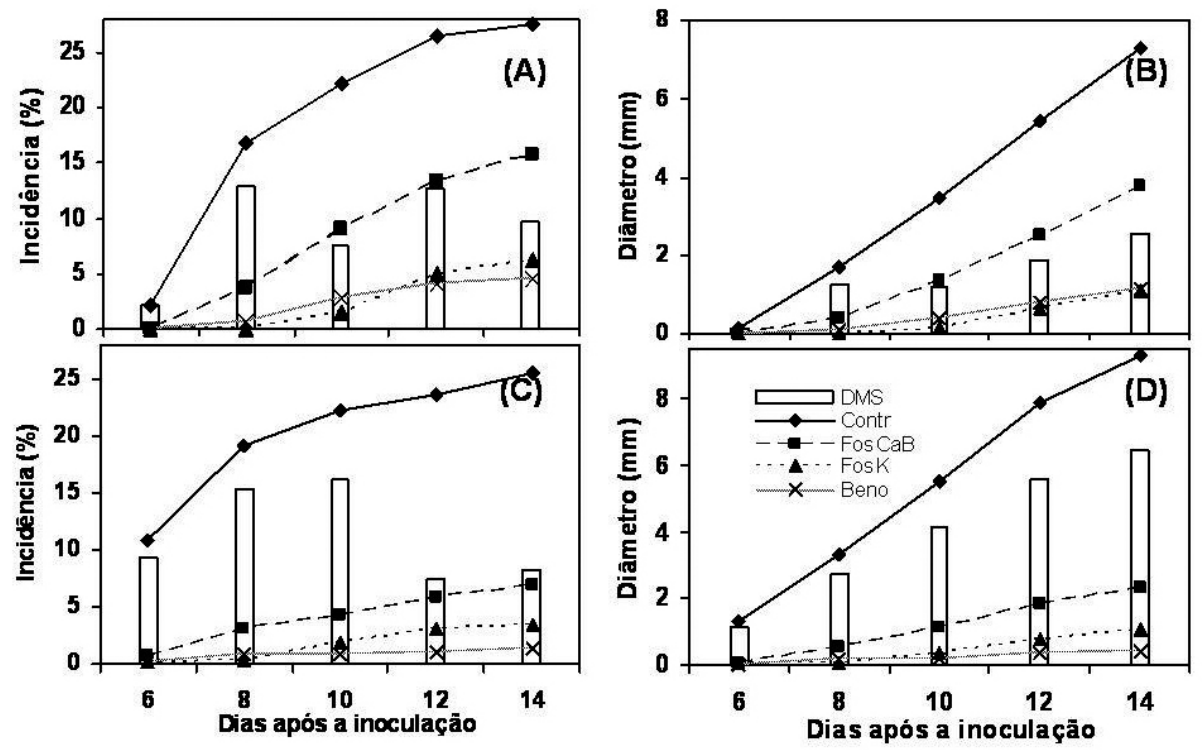

FIGURA 2 - Valores médios de incidência (\%) e diâmetro da lesão (mm) de mofo-azul, dos seis aos 14 dias após a inoculação de Penicillium expansum, em maçãs 'Fuji' (A e B) e 'Gala' (C e D) submetidas a diferentes tratamentos. Diferença minimamente significativa (DMS) entre tratamentos, calculada através do teste de Tukey $(\mathrm{P}<0,05)$, é representada pela barra branca. Tratamentos: Contr = testemunha somente com o patógeno; FosCaB = Fosfito-CaB $\left(1,5\right.$ ml. $\left.\mathrm{L}^{-1}\right)$; FosK = Fosfito-K $(1,5$ $\left.\mathrm{ml} . \mathrm{L}^{-1}\right) ;$ e Beno $=$ Benomil $\left(150 \mathrm{mg} \cdot \mathrm{L}^{-1}\right)$. Lages-SC, 2001-2002. 


\section{CONCLUSÕES}

O presente trabalho mostrou que os tratamentos póscolheita com fosfito-K $\left(0,75\right.$ a 1,5 mL. $\left.\mathrm{L}^{-1}\right)$ e fosfito-CaB $(1,5$ a 3,0 $\left.\mathrm{mL} . \mathrm{L}^{-1}\right)$ reduziram a incidência do mofo-azul em maçãs. $\mathrm{O}$ fosfito $\mathrm{K}\left(1,50 \mathrm{~mL} . \mathrm{L}^{-1}\right)$ foi tão eficiente quanto o fungicida tradicional não mais comercializado benomil (150 mg.L $\left.\mathrm{L}^{-1}\right)$, na redução do mofo-azul em maçãs, quando aplicado à água contaminada com Penicillium expansum.

\section{AGRADECIMENTOS}

Os autores agradecem ao Conselho Nacional de Desenvolvimento Científico e Tecnológico $(\mathrm{CNPq})$ pelo apoio financeiro parcial a este projeto.

\section{REFERÊNCIAS}

BLUM, L.E.B; AMARANTE, C.V.T.; VALDEBENITOSANHUEZA, R.M. Postharvest application of the yeast Cryptococcus laurentii reduces apple rots. Acta Horticulturae, Leuven, n. 682, v. 3, p. 2.109-2.113, 2005.

BLUM, L.E.B; AMARANTE, C.V.T.; VALDEBENITOSANHUEZA, R.M.; GUIMARÃES, L.S.; DEZANET, A.; HACK NETO, P. Cryptococcus laurentii aplicado em póscolheita reduz podridões em maçãs. Fitopatologia Brasileira, Brasília, v. 29, n. 4, p. 433-436, 2004.

BRACKMANN, A.; GIEHL, R.F.H.; SESTARI, I.; STEFFENS, C.A. Fosfitos para o controle de podridões pós-colheita em maçãs 'Fuji' durante o armazenamento refrigerado. Ciência Rural, Santa Maria, v. 34, n. 4, p. 1039-1042, 2004.

DAVIS, A.J.; SAY, M.; SNOW, A.J.; GRANT, B.R. Sensitivity of Fusarium oxysporum f. sp. cubense to phosphonate. Plant Pathology, Oxford, v. 43, n. 1, p. 200-205, 1994.

LIMA, G.; SPINA, A.M.; CASTORIA, R.; DECURTIS, F.; DECICCO, V. Integration of Biocontrol agents and food-grade additives for enhancing protection of stored apples from Penicillium expansum. Journal of Food Protection, Des Moines, v. 68, n. 10, p. 2.100-2.106, 2005.

McKAY, A.G.; FLOYD, R.M.; BOYD, C.J. Phosphonic acid compounds controls downy mildew (Peronospora parasitica) in cauliflower curds. Australian Journal of Experimental Agriculture, Collingwood, v. 32, n. 1, p. 127129, 1992.

PANICKER, S.; GANGADHARAN, K. Controlling downy mildew of maize caused by Peronosclerospora sorghi by foliar sprays of phosphonic acid compounds. Crop Protection, Amsterdam, v. 18, n. 2, p. 115-118, 1999.

REUVENI, M.; SHEGLOV, D.; COHEN, Y. Control of moldy-core decay in apple fruits by â-aminobutyric acids and potassium phosphates. Plant Disease, St. Paul, v. 87, n. 8, p. 933-936, 2003.

ROSENBERGER, D.A. Blue mold. In: JONES, A.L.; ALDWINCKLE, H.S. Compendium of apple and pear diseases. St. Paul: APS Press, 1990. p. 54-55.
SALA, F.C.; COSTA, C.P.; ECHER, M.M.; MARTINS, M.C.; BLAT, S.F. Phosphite effect on hot and sweet pepper reaction to Phytophthora capsici. Scientia Agricola, Piracicaba, v. 61, n. 5, p. 462-495, 2004.

SPEISER, B.; BERNER, A.; HASELI, A.; TAMM, L. Control of downy mildew of grapevine with potassium phosphonate: effectivity and phosphonate residues in wine. Biological Agriculture and Horticulture, Bicester, v. 17, n. 4, p. 305$312,1999$.

WILD, B.L.; WILSON, C.L.; WINLEY, E.L. Apple host defense reactions as affected by cycloheximide, phosphonate, and citrus green mould, Penicillium digitatum. ACIAR Proceedings Series, Collingwood, v. 80, p. 155-161, 1998. 